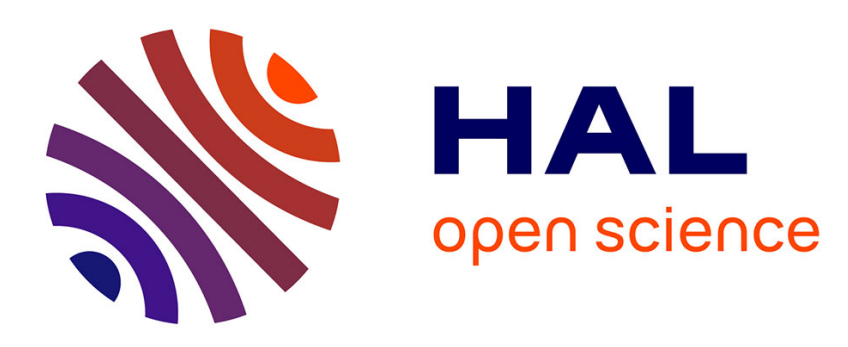

\title{
Davydov splitting and self-organization in a porphyrin layer noncovalently attached to Single wall carbon nanotubes
}

\author{
Géraud Delport, Fabien Vialla, Cyrielle Roquelet, Stéphane Campidelli, \\ Christophe Voisin, Jean-sébastien Lauret
}

\section{To cite this version:}

Géraud Delport, Fabien Vialla, Cyrielle Roquelet, Stéphane Campidelli, Christophe Voisin, et al.. Davydov splitting and self-organization in a porphyrin layer noncovalently attached to Single wall carbon nanotubes. Nano Letters, 2017, 17 (11), pp.6778 - 6782. 10.1021/acs.nanolett.7b02996 . cea-01630881

\section{HAL Id: cea-01630881 https://hal-cea.archives-ouvertes.fr/cea-01630881}

Submitted on 8 Oct 2021

HAL is a multi-disciplinary open access archive for the deposit and dissemination of scientific research documents, whether they are published or not. The documents may come from teaching and research institutions in France or abroad, or from public or private research centers.
L'archive ouverte pluridisciplinaire HAL, est destinée au dépôt et à la diffusion de documents scientifiques de niveau recherche, publiés ou non, émanant des établissements d'enseignement et de recherche français ou étrangers, des laboratoires publics ou privés. 


\title{
Davydov splitting and self-organization in a porphyrin layer noncovalently attached to single wall carbon nanotubes
}

\author{
Géraud Delport, ${ }^{\dagger}$ Fabien Vialla ${ }^{\ddagger}$ Cyrielle Roquelet, ${ }^{\dagger}$ Stéphane Campidelli, ${ }^{\boldsymbol{9}}$ \\ Christophe Voisin, ${ }^{\ddagger}$ and Jean-Sébastien Lauret ${ }^{*} \dagger$ \\ †Laboratoire Aimé Cotton, CNRS, Univ. Paris-Sud, ENS Cachan, Université Paris-Saclay, \\ 91405 Orsay Cedex \\ $\ddagger$ Laboratoire Pierre Aigrain, Ecole Normale Supérieure, CNRS, UPMC, Université Paris \\ Diderot, Paris, France \\ ฯLICSEN, NIMBE, CEA, CNRS, Université Paris-Saclay, CEA Saclay 91191 \\ Gif-sur-Yvette Cedex, France \\ E-mail: jean-sebastien.lauret@lac.u-psud.fr \\ Phone: +33-1-69352132
}

\begin{abstract}
We study the ability of porphyrin molecules to cooperate upon adsorption on the $\mathrm{sp}^{2}$ curved surface of carbon nanotube. We discuss the role of the phenyl substituents in the cooperativity of the functionalization reaction. Moreover, a specific spatial organization of the molecules around the nanotube is unveiled through polarization sensitive experiments. Furthermore, we observe an increase of the energy splitting of the porphyrin main transition upon the adsorption on the nanotube. This effect, interpreted as a Davydov splitting, is analyzed quantitatively using a dipole-dipole coupling model. This study demonstrates the ability of porphyrin molecules to create an organized selfassembled layer at the surface of the nanotubes where molecules are electronically coupled together.
\end{abstract}

\section{Keywords}

Self-assembly, Energy transfer, Hybrid nanomaterials, Functionalization, Davydov Splitting, Nanotube, Porphyrin
Over the years, the development of selfassembled nanomaterials has lead to major breakthroughs in various domains such as catalysis, ${ }^{1}$ electronics, ${ }^{2}$ or photonics. ${ }^{3}$ For instance, thin films made of self-assembled organic or hybrid materials are promising building blocks for optoelectronic devices, such as heterojunctions solar cells ${ }^{4}$ or organic LEDs. ${ }^{5}$ Self-assembled nanomaterials have remarkable light harvesting and charge transport properties, that are often connected to their degree of organization. ${ }^{6}$ Therefore, one key challenge in nanoscience is to understand and control the organization of nanomaterials at the supramolecular scale.

In this context, many groups have developed self-assembled nanocompounds based on carbon materials (nanotube, fullerene ...). ${ }^{9-17}$ In particular, our groups have evidenced an ultraefficient $(\sim 100 \%)$ energy transfer in compounds made of tetraphenylporphyrins (TPP) molecules (donor) and carbon nanotubes (acceptor). ${ }^{18-22}$ This effect is even more remarkable in the context of non-covalent functionalization. In addition, despite the weak $\pi$ stacking interaction these compounds show outstanding chemical stability in water. ${ }^{21,23}$ In a 


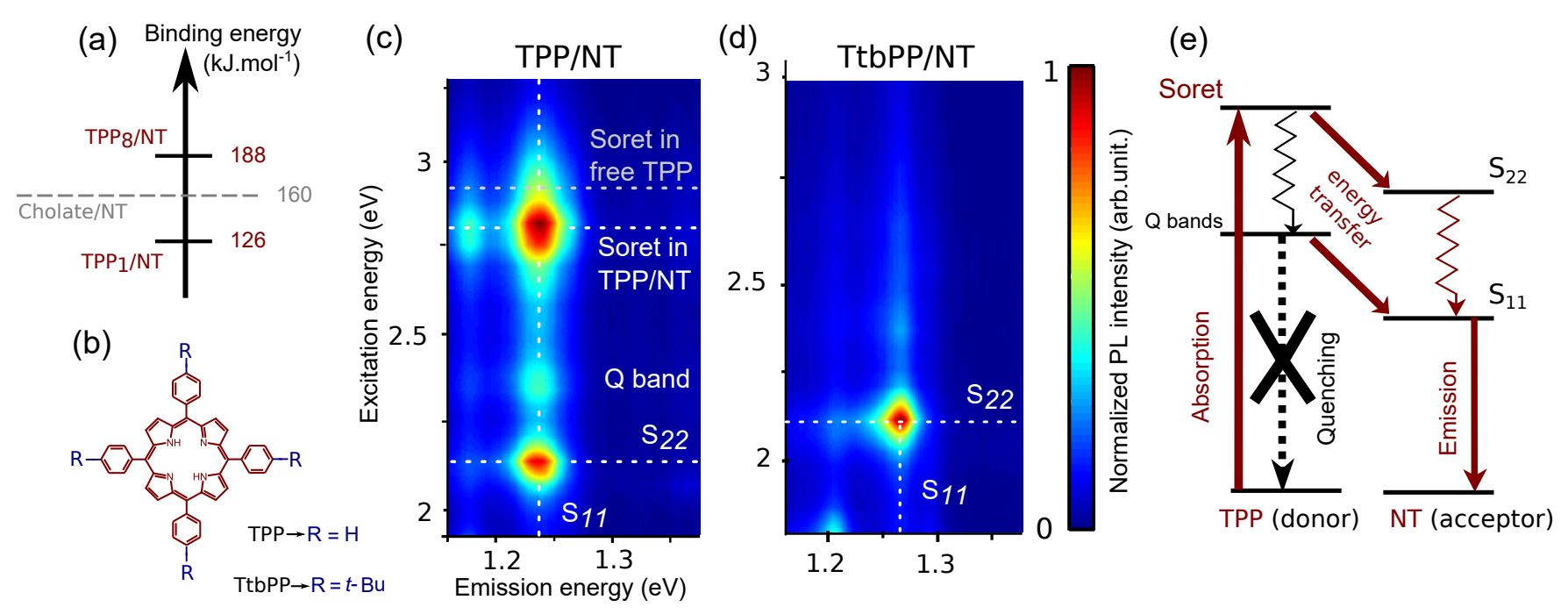

Figure 1: (a) Binding energies for TPP/NT systems (one and eight molecules) and cholate/NT systems as calculated in ${ }^{7}$ and; ${ }^{8}$ (b) Chemical representation of the 5,10,15,20-(tetraphenyl)-porphyrin (TPP) and the 5,10,15,20-(tetra-4-tert-butylphenyl)-porphyrin (TtbPP); PLE map of the TPP/NT sample (c) and the TtbPP/NT sample (d). (e) Scheme of the energy transfer process.

previous thermodynamics study, we demonstrated that the reaction is cooperative in the context of a Hill model. One possible origin of the cooperativity of the reaction lies in molecule-molecule interaction. ${ }^{24}$ Therefore, these observations raise two questions: the origin of the possible TPP-TPP interaction and the degree of organization of the molecules at the nanotube surface.

In this article, we show that porphyrin molecules self-assemble to form an organized shell around the nanotube. In particular, we show that the interaction between the phenyl substituents is central for the self-assembly. This effect has been investigated by modifying chemically the molecule. The degree of organization has been probed by using a polarized photoluminescence (PL) spectroscopy approach. Beside chemical stability, a spectroscopic signature of the interaction between TPP is a strong Davydov splitting resulting from a dipole-dipole interaction.

Concomitantly with our experimental investigations, some theoretical predictions on the organization of TPP around nanotubes have been reported. First, a recent DFT study from W. Orellana ${ }^{7}$ has predicted a cooperative behavior during the adsorption of TPP on nanotubes. He has calculated several TPP patterns at the surface of a $(6,5)$ nanotube
(NT). The most stable one turns to be a eight TPP building block, due to the phenyl-phenyl stabilizing interaction. A binding energy per molecule in the organized structure of $1.95 \mathrm{eV}$ (188 kJ.mol ${ }^{-1}$ ) was extracted, which is significantly larger than the $1.31 \mathrm{eV}\left(126 \mathrm{~kJ} . \mathrm{mol}^{-1}\right)$ value obtained for the adsorption of a single TPP. Thus, the cooperation of the porphyrins leads to a stabilization of the global TPP/NT system. The author attributed this effect to the stabilizing $\pi$-stacking interaction between the phenyl groups of adjacent porphyrins. ${ }^{25}$ Likewise, Bassiouk et al. have performed molecular mechanics simulations and have also predicted an organized structure of the TPP molecules around the nanotube. ${ }^{26}$

In our experiments, the functionalization is performed in an aqueous suspension with sodium cholate micelles. In this process, the porphyrins have to overcome the cholate/NT binding energy barrier to reach the NT surface. The binding energy of cholate on nanotubes have been calculated by Carvhalo et al. ${ }^{8}$ The value is of the order of $160 \mathrm{~kJ} \cdot \mathrm{mol}^{-1}$. It places the binding energy of cholate in between the one of a single TPP and the one of the eight TPP configuration reported by $\mathrm{W}$. Orellana (see figure 1(a)). Therefore, it suggests that the TPPTPP interaction is a key parameter to surpass the cholate/NT binding in order to enable the 
stacking of the TPP onto the NT surface.

To experimentally test this theory, we have functionalized $(6,5)$ NTs with two kinds of porphyrins using the micelle swelling method. ${ }^{21,27}$ The photoluminescence excitation (PLE) map of TPP/NT compounds is displayed in figure 1(c). In addition to the intrinsic $S_{22} \rightarrow S_{11}$ transition of the $(6,5) \mathrm{NTs}$, an additional resonance is observed for an excitation energy at $2.82 \mathrm{eV}$. This transition is a direct signature of the energy transfer from the TPPs to the nanotube. ${ }^{19}$ It corresponds to a photon absorption on the Soret band (B) of the porphyrins stacked on the NT, and an emission on the $\mathrm{S}_{11}$ excitonic transition of the NT. ${ }^{24}$ In a second sample, we have used modified porphyrins 5,10,15,20(tetra-4-tert-butylphenyl)-porphyrin (TtbPP). These molecules have tertbutyl substituents on the phenyl groups, that increase their steric hindrance (see figure 1(b)) and may prevent the phenyl-phenyl interaction. The PLE map of the final TtbPP/NT sample (figure 1 (d)) does not display any evidence of functionalization: no TtbPP $\rightarrow \mathrm{NT}$ energy transfer and no shift of the optical transitions. In principle, the blocking substituents should not hinder the interaction of the TPP macrocycle with the nanotube surface. Therefore, the absence of functionalization seems to be specifically related to the suppression of the phenyl-phenyl interaction induced by the steric hindrance of the tertbutyl, confirming the importance of such interaction to achieve chemically stable compounds. This strongly supports that the stacking reaction arises from small clusters of few TPP interacting together through the phenyl-phenyl interaction, as predicted by Orellana et al. ${ }^{7}$

This phenyl-phenyl interaction implies some ordering (at least local) of the TPP molecules at the surface of the nanotube. In the following, we look for spectroscopic evidences of such order, using polarized PLE (see figure S4 in the S.I. for details). For different excitation frequencies $\omega$, we have measured the $\mathrm{PL}$ anisotropy $r(\omega)$ (figure S5 in S.I.):

$$
r(\omega)=\frac{I_{V V}(\omega)-G(\omega) I_{V H}(\omega)}{I_{T}(\omega)}
$$

where $I_{V V}$ (resp. $I_{H H}$ ) is the $\mathrm{PL}$ intensity using two vertical (resp. horizontal) polarizers. $G(\omega)=I_{H V} / I_{H H}$ is a correction factor ${ }^{28}$ and $I_{T}(\omega)=I_{V V}(\omega)+2 G I_{V H}(\omega)$ is the total PL intensity. The anisotropy diagram (figure S5 in S.I.) allows us to determine the maximum of the anisotropy coefficient $r_{\max }$. This coefficient lies between 0.4 and -0.2 for any solution of randomly oriented luminescent objects, ${ }^{28}$ while the upper limit (resp. the lower) is obtained when these objects have fully parallel (resp. orthogonal) absorption and emission dipoles. For the TPP/NT suspension, $r_{\max }$ is here evaluated to 0.25 , in good agreement with the literature. $^{29}$ From $r(\omega)$, it is possible to extract $\mathrm{I}_{\|}$and $\mathrm{I}_{\perp}$. These PL intensities represent two different PL processes in which the excitation and emission dipoles are either aligned $\left(\mathrm{I}_{\|}\right)$or orthogonal $\left(\mathrm{I}_{\perp}\right):^{28}$

$$
\begin{gathered}
I_{\|}(\omega)=\frac{r(\omega)+0.5 \times r_{\max }}{1.5 \times r_{\max }} I_{T} \\
I_{\perp}(\omega)=\frac{r_{\max }-r(\omega)}{1.5 \times r_{\max }} I_{T}
\end{gathered}
$$

Figure 2(a) displays the $\mathrm{I}_{\|}$and $\mathrm{I}_{\perp}$ PLE contributions for the TPP/NT sample and for a detection energy fixed at $1.24 \mathrm{eV}\left(\mathrm{S}_{11}\right.$ of the $(6,5)$ chirality). In the $\mathrm{S}_{22}$ spectral region (at $2.1 \mathrm{eV}$ ), the signal is concentrated in the $I_{\|}$intensity, in agreement with the literature for pristine nanotubes samples in surfactants. ${ }^{29}$ It corresponds to the resonant absorption and emission of light polarized along the NT axis, in connection with the optical selection rules of nanotubes. ${ }^{30}$

In the Soret (B) region, through the energy transfer, the behavior is more complex, with two subbands located at different energies. A similar splitting has been evidenced in isolated TPP, ${ }^{31}$ and corresponds to the orthogonal Soret transitions $\mathrm{B}_{x}$ and $\mathrm{B}_{y}$ (see figure 2 (a) inset). The low energy one $\left(B_{x}\right)$ is here located at $2.82 \mathrm{eV}$. We see that it is concentrated in the $\mathrm{I}_{\|}$signal, proving that the TPP absorption dipole is aligned with the emission dipole of the NT. As this emission dipole is aligned with the $\mathrm{NT}$ axis, we can deduce that the $\mathrm{B}_{x}$ dipoles of the TPP are also along this direction. Therefore, this experiment shows that, on average, 

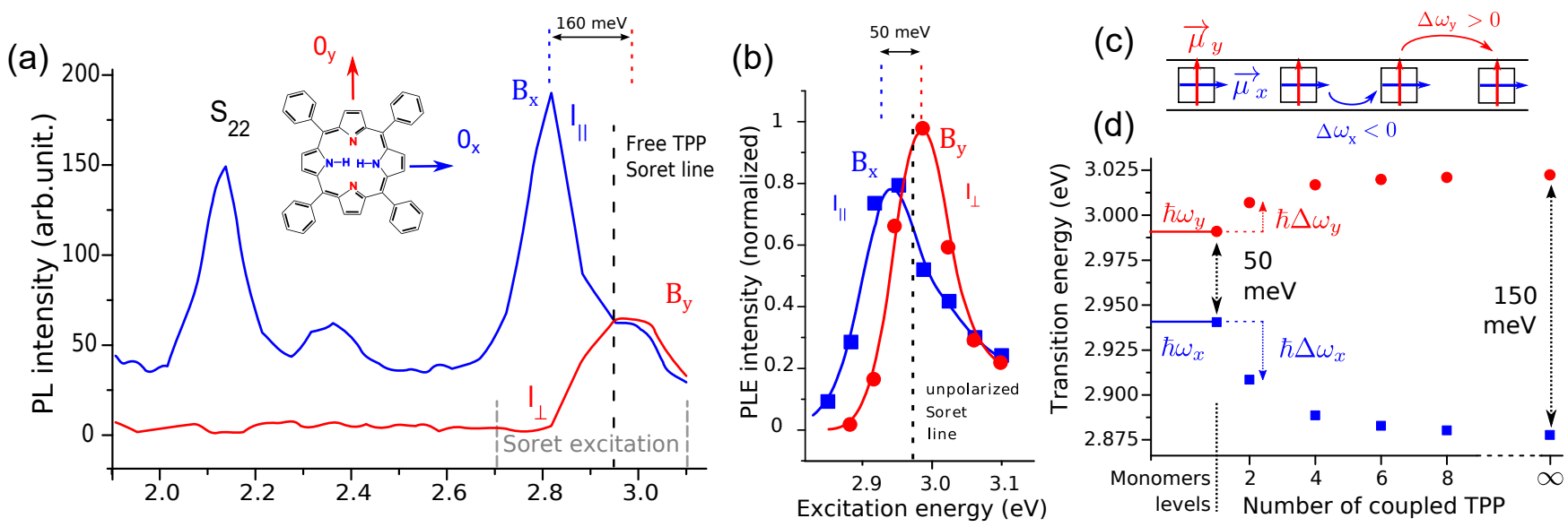

Figure 2: (a) $\mathrm{I}_{\|}$and $\mathrm{I}_{\perp}$ PLE components from a TPP/NT suspension, for an emission energy of $1.24 \mathrm{eV}$ (corresponding to the $(6,5)$ functionalized nanotubes). The maximum of the Soret absorption band at $2.952 \mathrm{eV}$ of the non interacting TPPs in cholate is marked with a vertical line. Inset: sketch of the molecule and definition of the axes. (b) $\mathrm{I}_{\|}$and $\mathrm{I}_{\perp} \mathrm{PLE}$ components within the Soret band of isolated TPP molecules (PL detected on the $\mathrm{Q}_{x}(0,0)$ band at $1.91 \mathrm{eV}$ ) in a DCM/castor oil mix. The maximum of the absorption Soret band of the TPPs at $2.967 \mathrm{eV}$ is shown with a vertical dashed line. (c) Schematic of an array of porphyrins at the surface of a carbon nanotube, with their $\mu_{x}$ dipoles aligned to the nanotube axis; (d) Evolution of the low energy $\hbar \omega_{x}$ and the high energy $\hbar \omega_{y}$ bands as a function of the number of porphyrin monomers in the aggregate.

all the TPP molecules share the same orientation with their $\mathrm{O}_{x}$ direction along the nanotube axis. This is the first optical evidence of an organization of the adsorbed TPPs on NTs.

In addition, this polarization diagram reveals the presence of the high energy subband $\mathrm{B}_{y}$ at $2.98 \mathrm{eV}$ in the $\mathrm{I}_{\perp}$ intensity. This is consistent with an orientation of the TPP molecules with their $\mathrm{O}_{y}$ direction perpendicular to the NT axis. Similar results were found for other chiralities in experiments on HiPCO NT samples (see figure S6). Interestingly, confocal PL experiments revealed that such effect is also present at the single TPP/NT nanocompound level (see figure S7). This proves that the alignment of TPP along the NT axis occurs in different experimental conditions, and that the compound is stable enough to keep its supramolecular structure upon deposition on a substrate.

The value of the $\mathrm{B}_{x} / \mathrm{B}_{y}$ energy splitting is of the order of $160 \mathrm{meV}$ in $\mathrm{TPP} / \mathrm{NT}$ compounds (see figure $2(\mathrm{a})$ ). To compare this splitting to the one of isolated TPP, we have performed a similar polarized PLE experiment on isolated $\mathrm{TPP}$ in a DCM/castor oil organic phase. The castor oil is a viscous solvent that is employed to slow down the rotational dynamics of TPPs below their PL lifetime, so that the rotational depolarization effect becomes negligible. ${ }^{32} \mathrm{~A} 10$ 90\% DCM-castor oil volume ratio was chosen to achieve such effect. Figure 2 (b) displays the polarized PLE signal of this TPP sample detected on the PL of the $\mathrm{Q}$ bands. The two orthogonal Soret components (see figure S9 in S.I. for the $\mathrm{Q}$ bands) of the porphyrins can be observed. The $\mathrm{B}_{x}$ resonance is observed at $2.94 \mathrm{eV}$ and the $\mathrm{B}_{y}$ one at $2.99 \mathrm{eV}$. Therefore, the $\mathrm{B}_{x} / \mathrm{B}_{y}$ splitting is of $50 \pm 10 \mathrm{meV}$ that is significantly smaller than the one found on the hybrid nanocompounds $(160 \pm 10 \mathrm{meV})$. Thus, the adsorption process on the NT clearly leads to an enhancement of the splitting between the $\mathrm{B}_{x}$ and $\mathrm{B}_{y}$ bands.

To determine the origin of these results, we have considered two main physical effects. The first one is the flattening of the molecule upon adsorption on a subtrate. ${ }^{33}$ Under such hypothesis, Chernia et $a l^{34}$ have predicted that the $\mathrm{B}_{x}$ and $\mathrm{B}_{y}$ bands would both undergo a similar redshift without an increase of the split- 
ting. In a calculation detailed in S.I., we have quantified that both bands undergo a redshift of $\sim-40 \mathrm{meV}$. As a conclusion, this flattening cannot explain the observed enhancement of the $\mathrm{B}_{x} / \mathrm{B}_{y}$ splitting. The other possible origin is an electronic coupling between organized TPP at the nanotube surface. Davydov splitting effects have been observed in aggregates of porphyrins under many different configurations, ${ }^{35-37}$ including adsorption on graphene. ${ }^{38,39}$ In this context, the respective red and blue shift could be the signature of an inequivalent dipole coupling for the $\mathrm{B}_{x}$ and $\mathrm{B}_{y}$ transitions. To model this effect, we have calculated the spectral shifts of the Soret band components within a linear aggregate of porphyrins as it could exist along the nanotube template (see figure $2(\mathrm{c})$ ). This configuration has been chosen for its simplicity. The case of a more realistic helical structure ${ }^{26}$ is treated in the SI. We have used Kasha's theory on coherent molecular aggregates ${ }^{40}$ In the following, the two components of the Soret band will be treated as orthogonal transition dipoles $\overrightarrow{\mu_{x}}$ and $\overrightarrow{\mu_{y}}$ (of amplitude $\mu_{0}=8.3 \mathrm{D}$, extracted from the TPP extinction coefficients, see figure S10 in S.I. for the details of the evaluation). This allows to evaluate the coupling term $\hbar \Delta \omega$ between two parallel dipoles:

$$
\hbar \Delta \omega=\frac{\mu_{0}^{2}}{4 \pi \varepsilon} \frac{1-3 \cos ^{2}(\theta)}{r^{3}}
$$

where $r$ is the distance between the dipoles and $\theta$ is the angle between the dipoles and the axis connecting the two dipoles. Starting from the initial transition energy $\omega_{0}$ of the two coupled dipoles, it leads to a final transition energy of $\hbar \omega_{\text {dimer }}=\hbar \omega_{0}+\hbar \Delta \omega$. Based on the results from figure 2 (a), we will consider that every TPP has its $x$ direction aligned with the nanotube axis. We will also assume a distance of $r=1.4 \mathrm{~nm}$ between neighbour molecules (the $\mathrm{TPP}$ diameter is $\simeq 1.25 \mathrm{~nm}$ ), as it was measured by STM for similar TPP aggregates on graphene. ${ }^{41}$ The numerical value of the coupling term $\hbar \Delta \omega$ is then evaluated for the $\overrightarrow{\mu_{x}}(\theta=0)$ and $\overrightarrow{\mu_{y}}(\theta=\pi / 2)$ set of dipoles. This yields respectively $\hbar \Delta \omega_{x}=-32 \mathrm{meV}$ (redshift) and $\hbar \Delta \omega_{y}=+16 \mathrm{meV}$ (blueshift).

Then, we evaluate the energy position of the two optical transitions $\omega_{x}$ and $\omega_{y}$, as a function of the number of coupled porphyrins (figure 2 (d)). Starting from the intrinsic $50 \pm 10 \mathrm{meV}$ found in castor oil, the energy splitting varies from $98 \pm 10 \mathrm{meV}$ for the dimer case up to $150 \pm 10 \mathrm{meV}$ for aggregates of 8 monomers or more. This final value has the same order of magnitude as the experimental splitting reported in figure 2(a) on TPP/NT complexes $(160 \pm 10 \mathrm{meV})$. Therefore, this dipole-dipole coupling model for a linear chain of TPP is able to explain the observed increase of the Soret energy splitting for TPP/NT samples. Note here that the Davydov splitting saturates for $\sim 8$ coupled monomers. This corresponds also to the number of TPP molecules in a cluster for which the best stability of the hybrid compound has been predicted. ${ }^{7}$ In this framework, no variation of the Davydov splitting is expected as a function of the concentration, and even the first small cluster stacked on the nanotube should show similar $\mathrm{B}_{x} / \mathrm{B}_{y}$ splitting to the one of the fully covered compound. Moreover, as shown in S.I., this model also provides similar energy splittings for helical aggregates that were also predicted recently. ${ }^{26}$

Finally, we investigate the reason why the $\mathrm{B}_{y}$ resonance is four times weaker than the $\mathrm{B}_{x}$ one in the TPP/NT samples. Indeed, this is in contrast with the situation in isolated TPP, where the $\mathrm{B}_{x}$ and the $\mathrm{B}_{y}$ transitions have similar amplitudes (see figure 2 (a) and (b)). This result can be interpreted as a consequence of a local field effect at the surface of the NT. As explained in a previous report (see also in the SI), an incoming electric field with a polarization perpendicular to the NT axis will have a locally screened amplitude, while no screening occurs for a parallel electric field. ${ }^{42}$ Consequently, using the data reported earlier, ${ }^{42}$ we evaluate a reduction by a factor four of the number of photons absorbed on the $\mathrm{B}_{y}$ transition (orthogonal to the NT axis) as compared to the $\mathrm{B}_{x}$ one (parallel to the NT axis).

We end up with a full model able to reproduce quantitatively the polarized spectra obtained on the NT/TPP samples. The inputs of the model consist in the data obtained on isolated TPP in castor oil (see figure 2(b)). Tak- 


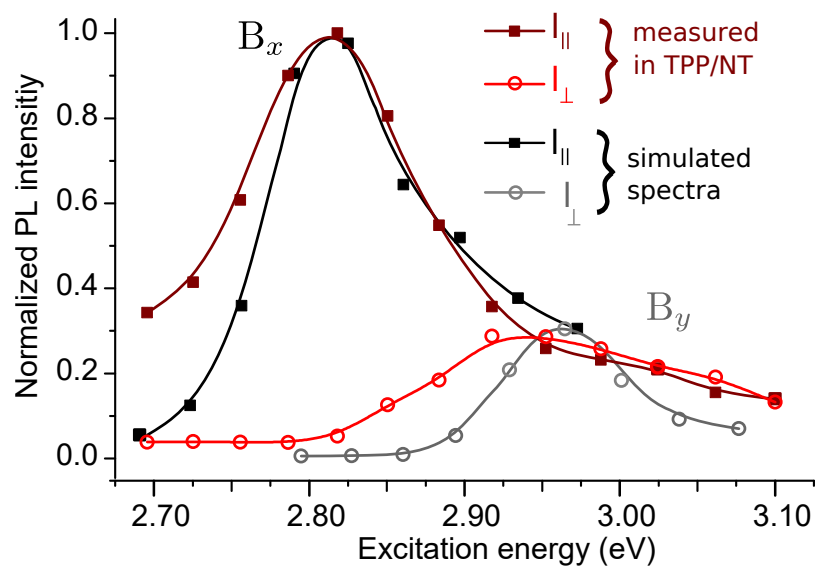

Figure 3: Comparison between the measured $\mathrm{I}_{\|}$ and $I_{\perp}$ PLE intensities for NT/TPP and the full model. The inputs of the model are the data obtained on isolated TPPs in castor oil.

ing into account the effects described above, we obtain the simulated spectra described on figure 3 (see figure S15 for the detail of each step). It reproduces both the energy position of the lines and their relative amplitude, validating our analysis. Nevertheless, the measured resonances have larger width than the simulated ones. This can be due to some disorder in the porphyrin layer (different angles, distances, ...) leading to a weak dispersion in the energy splitting. Changes in width can also be explained by vibronic coupling effects within the aggregates ${ }^{43}$ that have been neglected in the present model.

Finally, we end up with a scheme where the stacking unit is composed of at least eight TPP molecules that are interacting together through the phenyl-phenyl interaction. In all clusters, each TPP molecule orientates its $\mathrm{O}_{x}$ direction along the nanotube axis. In these aggregates, the molecule-molecule distance is small leading to a dipole-dipole coupling which signature is the observed Davydov splitting.

\section{Conclusion}

We carried out a comprehensive optical study of the cooperative stacking of porphyrin molecules onto carbon nanotubes. First of all, the role played by the phenyl groups in the organization process has been demonstrated experimentally by modifying their chemical structure. Moreover, we have shown through polarization sensitive experiments that the TPP molecules selforganize in a specific way with their $\mathrm{O}_{x}$ direction along the nanotube axis. This supramolecular organization leads to an electronic coupling between the molecules stacked onto the nanotube. A dipole-dipole model applied to this structure predicts an enhancement of the natural $\mathrm{B}_{x} / \mathrm{B}_{y}$ splitting of the molecule. This prediction has been confirmed experimentally by means of polarization resolved PLE experiments. Finally, our model, taking account all the physical effects (screening, flattening of the molecule, Davydov splitting) allows to reproduce the experimental data. It represents a significant advance in the understanding of the supramolecular organization of TPP molecules around nanotubes.

\section{Supporting Information Avail- able}

The following files are available free of charge.

Acknowledgement J.S.L. is partially funded by "Institut Universitaire de France".

\section{References}

(1) Daniel, M.-C.; Astruc, D. Chemical reviews 2004, 104, 293-346.

(2) Yu, D.; Dai, L. The Journal of Physical Chemistry Letters 2009, 1, 467-470.

(3) Medintz, I. L.; Clapp, A. R.; Mattoussi, H.; Goldman, E. R.; Fisher, B.; Mauro, J. M. Nature materials 2003, 2, 630-638.

(4) Pivrikas, A.; Sariciftci, N. S.; Juška, G.; Österbacka, R. Progress in Photovoltaics: Research and Applications 2007, 15, 677696.

(5) Baldo, M. A.; O’brien, D.; You, Y.; Shoustikov, A.; Sibley, S.; Thompson, M.; Forrest, S. Nature 1998, 395, 151-154. 
(6) Thorsmølle, V. K.; Averitt, R. D.; Demsar, J.; Smith, D.; Tretiak, S.; Martin, R.; Chi, X.; Crone, B.; Ramirez, A.; Taylor, A. Physical Review Letters 2009, 102, 017401.

(7) Orellana, W. Chemical Physics Letters 2015, 634, 47-52.

(8) Carvalho, E. J.; dos Santos, M. C. ACS nano 2010, 4, 765-770.

(9) Guldi, D. M. Chemical Communications 2000, 321-327.

(10) Wang, Y.; Li, Z.; Wang, J.; Li, J.; Lin, Y. Trends in biotechnology 2011, 29, 205212.

(11) Alvarez, L.; Almadori, Y.; Arenal, R.; Babaa, R.; Michel, T.; Le Parc, R.; Bantignies, J.-L.; Jousselme, B.; Palacin, S.; Hermet, P. The Journal of Physical Chemistry $C$ 2011, 115, 11898-11905.

(12) Ernst, F.; Heek, T.; Setaro, A.; Haag, R.; Reich, S. Advanced Functional Materials 2012, 22, 3921-3926.

(13) Hadj, K. E.; Bertoncini, P.; Chauvet, O. ACS Nano 2013, 8743-8752.

(14) Chen, Y.; Royal, G.; Flahaut, E.; Cobo, S.; Bouchiat, V.; Marty, L.; Bendiab, N. Advanced Materials 2017,

(15) Gaufres, E.; NTang, Y. W.; Lapointe, F.; Cabana, J.; Nadon, M. A.; Cottenye, F., N Raymond; Szkopek, T.; Martel, R. Nature Photonics 2014, 8, 72.

(16) Cambré, S.; Campo, J.; Beirnaert, C.; Verlackt, C.; Cool, P.; Wenseleers, W. Nature nanotechnology 2015, 10, 248-252.

(17) Gaufres, E.; Tang, N. Y.-W.; Favron, A.; Allard, C.; Lapointe, F.; Jourdain, V.; Tahir, S.; Brosseau, C.-N.; Leonelli, R.; Martel, R. ACS Nano 2016, 10, 1022010226.
(18) Magadur, G.; Lauret, J.-S.; AlainRizzo, V.; Voisin, C.; Roussignol, P.; Deleporte, E.; Delaire, J. A. ChemPhysChem 2008, 9, 1250-1253.

(19) Roquelet, C.; Garrot, D.; Lauret, J.S.; Voisin, C.; Alain-Rizzo, V.; Roussignol, P.; Delaire, J.; Deleporte, E. Applied Physics Letters 2010, 97, 141918.

(20) Clavé, G. et al. Chemistry of Materials 2013, 25, 2700-2707.

(21) Roquelet, C.; Lauret, J.-S.; AlainRizzo, V.; Voisin, C.; Fleurier, R.; Delarue, M.; Garrot, D.; Loiseau, A.; Roussignol, P.; Delaire, J. A. ChemPhysChem 2010, 11, 1667-1672.

(22) Vialla, F.; Roquelet, C.; Langlois, B.; Delport, G.; Santos, S. M.; Deleporte, E.; Roussignol, P.; Delalande, C.; Voisin, C.; Lauret, J.-S. Physical review letters 2013, 111, 137402 .

(23) Delport, G.; Orcin-Chaix, L.; Campidelli, S.; Voisin, C.; Lauret, J.-S. Nanoscale 2017, 9, 2646.

(24) Vialla, F.; Delport, G.; Chassagneux, Y.; Roussignol, P.; Lauret, J.-S.; Voisin, C. Nanoscale 2016, 8, 2326-2332.

(25) Orellana, W. Applied Physics Letters 2014, 105, 023110.

(26) Bassiouk, M.; Basiuk, V. A.; Basiuk, E. V.; Álvarez-Zauco, E.; MartínezHerrera, M.; Rojas-Aguilar, A.; PuenteLee, I. Applied Surface Science 2013, 275, $168-177$.

(27) Wang, R. K.; Chen, W.-C.; Campos, D. K.; Ziegler, K. J. Journal of the American Chemical Society 2008, 130, 16330-16337.

(28) Lakowicz, J. R.; Szmacinski, H.; Nowaczyk, K.; Berndt, K. W.; Johnson, M. Analytical biochemistry 1992, 202, 316-330. 
(29) Miyauchi, Y.; Oba, M.; Maruyama, S. Physical Review B 2006, 74, 205440.

(30) Lefebvre, J.; Finnie, P. Physical review letters 2007, 98, 167406.

(31) Weigl, J. W. Journal of Molecular Spectroscopy 1957, 1, 133-138.

(32) Lakowicz, J. R. Principles of fluorescence spectroscopy; Springer Science \& Business Media, 2013.

(33) Xu, Y.; Zhao, L.; Bai, H.; Hong, W.; Li, C.; Shi, G. Journal of the American Chemical Society 2009, 131, 1349013497.

(34) Chernia, Z.; Gill, D. Langmuir 1999, 15, 1625-1633.

(35) El-Nahass, M.; Zeyada, H.; Aziz, M.; Makhlouf, M. Spectrochimica Acta Part A: Molecular and Biomolecular Spectroscopy 2005, 61, 3026-3031.

(36) Vlaming, S.; Augulis, R.; Stuart, M.; Knoester, J.; Van Loosdrecht, P. The Journal of Physical Chemistry B 2009, 113, 2273-2283.

(37) Gibbs, E. J.; Tinoco, I.; Maestre, M. F.; Ellinas, P. A.; Pasternack, R. F. Biochemical and biophysical research communications 1988, 15\%, 350-358.

(38) Guo, P.; Chen, P.; Liu, M. ACS applied materials \& interfaces 2013, 5, 53365345 .

(39) Bussetti, G.; Campione, M.; Ferraro, L.; Raimondo, L.; Bonanni, B.; Goletti, C.; Palummo, M.; Hogan, C.; Duò, L.; Finazzi, M.; Sassella, A. The Journal of Physical Chemistry $C$ 2014, 118, 1564915655.

(40) Kasha, M.; Rawls, H.; Ashraf ElBayoumi, M. Pure and Applied Chemistry 1965, 11, 371-392.
(41) Pham, V. D.; Lagoute, J.; Mouhoub, O.; Joucken, F.; Repain, V.; Chacon, C.; Bellec, A.; Girard, Y.; Rousset, S. ACS nano 2014, 8, 9403-9409.

(42) Roquelet, C.; Vialla, F.; Diederichs, C.; Roussignol, P.; Delalande, C.; Deleporte, E.; Lauret, J.-S.; Voisin, C. ACS nano 2012, 6, 8796-8802.

(43) Roden, J.; Strunz, W. T.; Eisfeld, A. International Journal of Modern Physics B 2010, 24, 5060-5067. 


\section{Graphical TOC Entry}

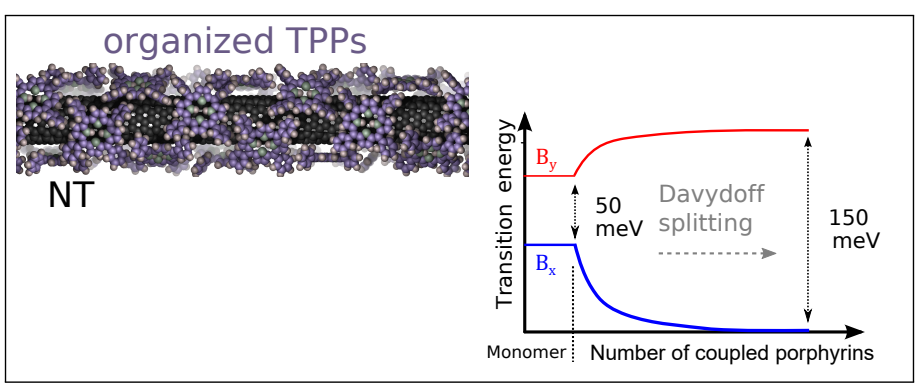

